\title{
Discussion on the Application of Warm Yang to Promote Qi Transformation in Acne Mastitis from "Yang Promotes Qi Transformation and Yin Promotes Forming"
}

\author{
Yupei Chen, Zhenlin Chen*, Pu Zhang \\ Shaanxi University of Chinese Medicine, Xianyang 712046, Shaanxi Province, China \\ *Corresponding author: Prof. Zhenlin Chen, chenzhenlin1997@163.com
}

\begin{abstract}
Yang promotes Qi transformation and Yin promotes forming" maintains the normal progress of human life activities, which is an important embodiment of human vitality. The dysfunction of "Yang promotes Qi transformation and Yin promotes forming" leads to the coagulation of pathological products such as phlegm and blood stasis in the breast meridian, resulting in the occurrence of acne mastitis. In clinical treatment, we should take "warm Yang to promote Qi transformation" as the treatment principle, and add or subtract according to the specific situation.
\end{abstract}

Keywords: Yang promotes Qi transformation and Yin promotes formation; Warm Yang to promote Qi transformation; Acne mastitis

Publication date: November 2021; Online publication: November 30, 2021

\section{Introduction}

Acne mastitis is a chronic non-bacterial disease based on mammary duct dilatation and plasma cell infiltration in non-lactation or menopause body ${ }^{[1]}$. Clinically, it is mainly manifested as breast mass, nipple overflow or even shortening depression, swelling and pain of axillary lymph nodes on the same side of the diseased breast, which is equivalent to plasma cell mastitis in western medicine. Due to the acne like contents can be seen after its ulceration, it is called acne mastitis ${ }^{[2]}$. The treatment is mainly surgery, which is easy to repeat after operation, and the postoperative wound is easy to form fistula, which cannot be healed and brings great pain to the patient's daily life ${ }^{[3]}$.

2. "Yang promotes Qi transformation and Yin promotes forming" to maintain the normal operation of life activities

"Yang turns into Qi and Yin forms" comes from "the Qi of clear Yang gathers on the top and becomes the sky, while the Qi of turbid Yin accumulates on the bottom and becomes the earth. Yin is more static, Yang is more restless: Yang governs generation, Yin governs growth: Yang governs suppression, Yin governs collection. Yang promotes Qi transformation and Yin promotes formation. "Yang Qi is warm, and the essence of visible Yin is transformed into Qi, which promotes all kinds of physiological activities of the viscera and limbs of human body. The invisible Qi is also restricted by Yin Qi, which generates fine substances such as blood essence and nourishes the viscera, holes and orifices. "Yin is calm and Yang is sound, so the spirit is cured. Yin and Yang restrict each other, coordinate and balance, so that all life activities of the human body can be carried out in an orderly manner. The functional state of "Yang promotes Qi transformation and Yin promotes formation" will also change with the change of human life 
vitality. With the gradual aging of the body or the induction of various internal and external disease factors, the relationship between the opposing constraints and the balance of growth and decline between Yin and Yang is disrupted, and the function of "Yang promotes Qi transformation and Yin promotes formation" is dysfunctional, so various diseases will also appear in the body.

The imbalance of the function of "Yang promotes Qi transformation and Yin promotes formation" can be divided into the following two aspects. If Yang Qi is hurt due to external infection with cold and damp or inner production of pathogen, "Yang transforming into Qi" is late, loses warmth and ability to grow, also fine substances cannot be transpired, gasified and dispersed in the whole body. Viscera and orifices lose nourishment, and "Yin becoming form" is relatively too much. Excessive convergence, condensation, so that Yin essence gathers somewhere to form pathological products such as phlegm, water dampness and blood stasis. Then, the formation of these pathological products also aggravates the cycle of "Yang transforming Qi," which leads to the delay of the disease. If the body feels heaty and the function of "Yang transforming into Qi" is too much, the body will show a heat image, which will also hurt the Yin essence over time. The "Yin becoming form" will lose its material basis, and all limbs and bones will lose their moistening, resulting in a series of Yin injury symptoms, such as feeling thirsty and excessive drinking, thin body, vexing heat in the chest, palms and soles, etc.

\section{3. "Yang promotes Qi transformation and Yin promotes forming" and acne mastitis}

"Treatment is based on origin, and origin is Yin and Yang." Yin and yang theory is widely used to explain the occurrence and development of various clinical diseases and guide the treatment of diseases. "Male nipples belong to the liver and breast belongs to the kidney; The female nipple belongs to the liver, and the breast belongs to the stomach." The occurrence of breast diseases is mainly related to the liver, kidney, spleen and stomach. These changes of visceral functions are inseparable from the mutual checks and balances of Yin and Yang. The imbalance of the function of "Yang promotes Qi transformation and Yin promotes formation" results in the accumulation of pathological products to form diseases. Among Yin and Yang, more emphasis is placed on the importance of Yang Qi to life activities, "once the sun shines, the haze disappears."

Liver Qi takes the initiative to coordinate the rise and fall of the whole body's Qi machine. Only when the Qi of the whole body is unobstructed and the liver can normally play the physiological function of "liver storing blood." If the function of "Yang transforming into Qi" is not enough, the liver Qi loses its regulation, and the liver storing blood loses its regulation, there will be nipple bleeding, even fluid overflow, metrorrhagia and metrostaxis, and the liver blood cannot moisten the tendons and eyes, showing symptoms such as dry eyes and urgent limbs. "Yang is insufficient to transform into Qi," and Qi is out of order, showing discomfort such as breast swelling and pain, chest tightness and shortness of breath. If Qi is not available, blood is also stagnant, liver wood invades the soil, water dampness overflows, resulting in phlegm dampness, and Qi stagnation, phlegm and blood stasis condenses in the breast meridian as a mass. If "Yang turns into Qi" and the liver rises too much, there will be chest, flank, breast and collateral pain, discomfort, fever, hyperactivity of liver wood, liver blood regulation loss, and a series of bleeding symptoms. The kidney governs water and Qi. If the "Yang transform into Qi" is less than that, the water and fluid will be lost in distribution and excretion, and the inability to absorb Qi will lead to excessive "Yin formation," and the formation of pathological products such as water dampness will also hinder the metabolism of water, fluid and Qi. The Yin and Yang of the kidney is the foundation of the Yin and Yang of the whole body. If the Yin and Yang function of the kidney is out of balance, the functions of all viscera cannot operate normally. The spleen and stomach are the foundation of the day after tomorrow. The spleen governs the transportation of food and water. If the function of "Yang transforming into Qi" is not enough, the spleen is unable to convert food into fine substances, or the essence is unable to spread throughout the 
body, abdominal distension and weight loss will occur. "Lack of Qi is cold." Without energy to transport water, water dampness and phlegm will condense in the weakest part of Yang Qi and become a mass. Deficiency of Qi and failing to control blood make the blood escape outside the pulse, and bleeding signs can also be seen. In the later stage of the disease or after operation, the wounds are often unable to nourish due to the insufficient function of "Yang transforming into Qi," the weakness of spleen transportation, and the delicacy of Qi and blood. The granulation of the wounds is bleak, luxuriance is loss, and the pus is dripping. This is "the source of deficiency of spleen and stomach, which is deficiency of Yang Qi and surplus of Yin Qi."

\section{4. "Warm Yang to promote Qi transformation" to treat acne mastitis}

In the Collection of Surgical Prescriptions: "For those who suffer from breast pain, they attack poison gas internally and feel wind evil externally, pour it into the blood vessels and send it in the breast, gradually become swollen and hard, blood coagulation and Qi stagnation or milk retention, and form welling and flat abscesses after a long time." In the early stage of acne mastitis, because the "Yang transforms into Qi" is not enough, the "Yin becomes form" is too much, and the pathological products such as Qi stagnation, blood stasis, phlegm coagulation and so on are bound in the breast meridians and become lumps. "The block is a tangible thing, which is formed by phlegm, food accumulation and dead blood [4]." The visible evil belongs to Yin. "Those who have disease and phlegm drink should be controlled by warming medicine," that is, warming Yang and promoting Qi transformation is the general principle for the treatment of visible evil. In the later stage, because the function of "Yang transforming into Qi" is too harmful to Yin, or the function of "Yang transforming into Qi" is not enough, it is unable to promote "Yin for the formation," the wound loses the nourishment of Yin essence and does not close for a long time. Therefore, in the clinical treatment of acne mastitis, we should "warm Yang to promote Qi transformation" from beginning to end, "carefully check the location of Yin and Yang and take harmony as the expect," so as to make the normal operation of Qi, blood and essence, eliminate the pathological products such as Qi stagnation, blood stasis and phlegm coagulation, and the postoperative wound can be nourished by Yin and essence, which makes wound heal quickly and prevent recurrence.

\section{Summary}

"Yang promotes Qi transformation and Yin promotes the formation" this maintains the normal operation of life activities. The pathogenesis of acne mastitis is due to the dysfunction and the stagnation of pathological products in the breast meridians. In treatment, we should take "Warming Yang and promoting Qi transformation" as the basic principle and adjust Yin and Yang to a balanced state. At the same time, we should carefully identify the stage of the disease and flexibly add and subtract in order to obtain good results.

\section{Disclosure statement}

The authors declare no conflict of interest.

\section{References}

[1] Lu M, Huang X, Zhan S, 2010, Analysis of MRI Signs of Plasma Cell Mastitis. Radiologic Practice, 25(6): 638-641.

[2] Liu D, Li Z, Yang M, et al., 2016, Analysis and Comparison of Two Digital Diagnostic Methods for 51 Cases of Acne Mastitis. Journal of Hunan University of Chinese Medicine, (4): 87-90.

[3] Yan X, 2018, Analysis of Treatment and Preventive Health Care Measures for Common Gynecological 
Diseases. World Latest Medicine Information, 18(79): $218+220$.

[4] Li G, 2014, Professor Tie Li's Experience in Clinical Application of Blood Activating Traditional Chinese Medicine. China Association of Chinese Medicine. Proceedings of the Second Qihuang Forum of China Association of Chinese Medicine - Collection of The Sub-Forum on TCM Prevention and Treatment of Blood Diseases. China Association of Chinese Medicine: Blood Diseases Branch of China Association of Chinese Medicine, 574-577. 